\title{
Renorrhaphy Using Pledgeted Sutures for Grade IV Renal Trauma
}

\author{
Dong Hun Kim \\ Department of Trauma Surgery, Trauma Center, Dankook University Hospital, Cheonan, Republic of Korea
}

\begin{abstract}
Although the overall trend is obviously toward less-invasive interventions, patients with low-grade injuries (grades I-III) are overwhelmingly managed conservatively, and those with high-grade injuries are most probably undergoing surgical treatment. Surgical treatment for grade IV renal injury according to the injury scale of the American Association for the Surgery of Trauma with unstable hemodynamics will probably lead to renal salvage than nephrectomy. The success of conservative surgery is based on early vascular control and various reconstructive renorrhaphy techniques. Here, we have described a successful renorrhaphy using pledgeted sutures for grade IV renal laceration after blunt trauma in a 56-year-old male.
\end{abstract}

(Trauma Image Proced 2018(2):65-67)

Key Words: Renorrhaphy; Kidney; Treatment; Trauma

\section{CASE}

The wire under construction hit the right back of a 56-year-old male (Fig. 1). Upon admission, the patient's blood pressure was $92 / 59 \mathrm{~mm} \mathrm{Hg}$, pulse rate was 125 beats/min, and he had been transfused with 2 units of packed red blood cell. The focused assessment with sonography for trauma revealed intra-abdominal fluid collection in the Morison pouch. Abdomen computed tomography (CT) performed in response to resuscitation demonstrated multiple laceration at the Couinaud segment V, VI, and VII of the liver, and multiple lacerations deep to the calyx in the right kidney with the contrast extravasation (Fig. 2). The injury severity score was 26. Therefore, he underwent emergency laparotomy due to intermittent hypotension. Right renorrhaphy with temporary renal vascular pedicle clamping after the

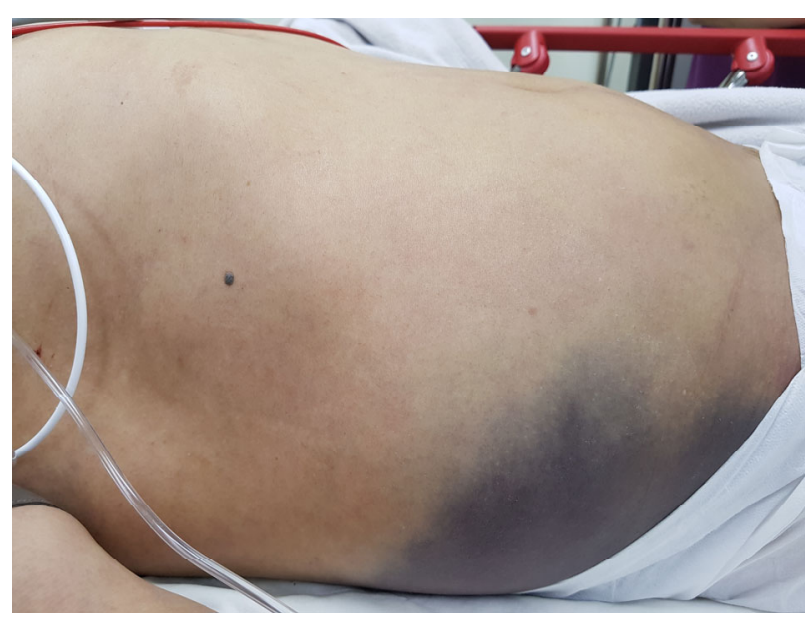

Fig. 1. External photographs revealing soft tissue bruising in the right back and flank.

temporary perihepatic packing was performed with transfusion of 2 units of packed red blood cell during

Received: October 8, 2018 Revised: November 1, 2018 Accepted: November 1, 2018

Correspondence to: Dong Hun Kim, Department of Trauma Surgery, Trauma Center, Dankook University Hospital, 201 Manghyang-ro, Dongnam-gu, Cheonan 31116, Republic of Korea

Tel: 82-41-550-7661, Fax: 82-41-550-0039, E-mail: saint7331@gmail.com

Copyright (c) 2018 Korean Association for Research, Procedures and Education on Trauma. All rights reserved.

@This is an open-access article distributed under the terms of the Creative Commons Attribution Non-Commercial License (http://creativecommons.org/ licenses/by-nc/4.0) which permits unrestricted noncommercial use, distribution, and reproduction in any medium, provided the original work is properly cited 

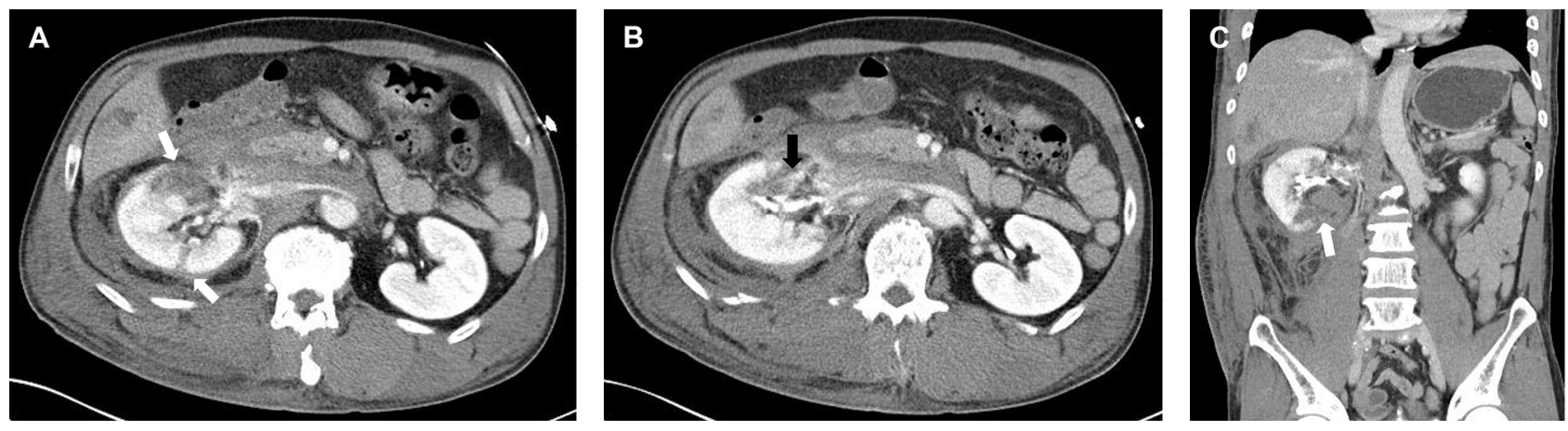

Fig. 2. An abdominal computed tomography shows multiple parenchymal lacerations and perirenal hematoma extending to the retropancreatic space (white arrow), and contrast extravasation from the lacerated renal calyx (indicated by white arrow). (A) and (B) Axial view, (C) Coronal view
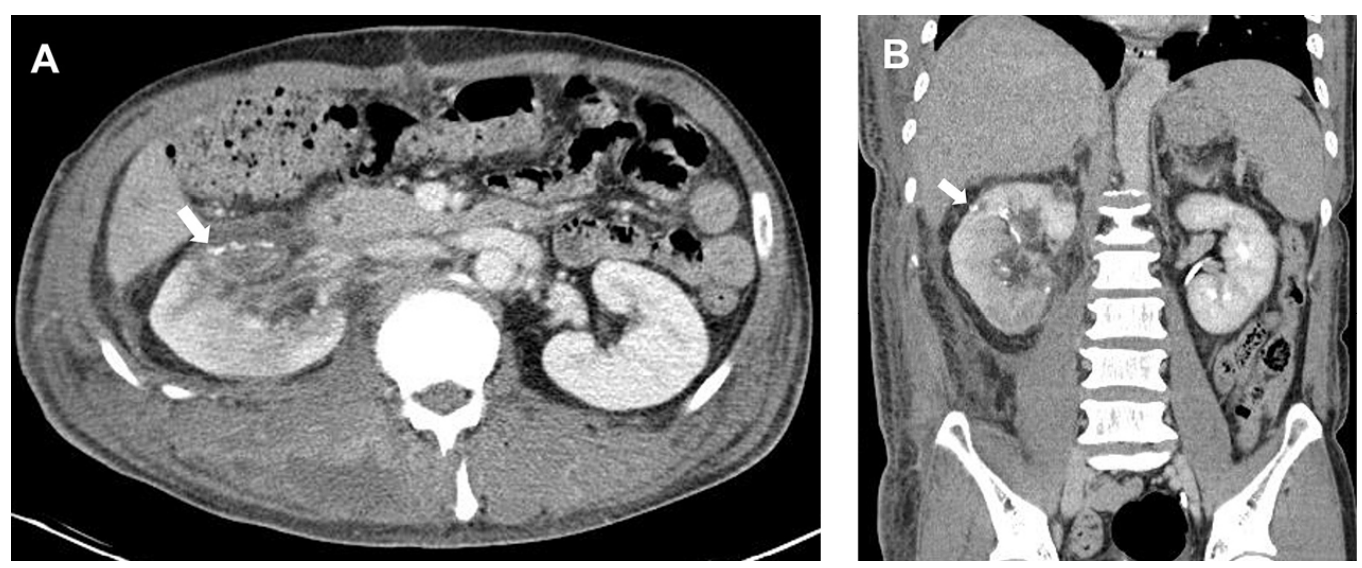

Fig. 3. A follow-up abdominal computed tomography on postoperative day 8 showing primary repair sites with pledgeted sutures (white arrow) on the lacerated renal parenchyma and perirenal hematoma resorption.

(A) Axial view, (C) Coronal view

the operation time of 165 min (Video 1), with the clamping time of $20 \mathrm{~min}$ at the right vascular pedicle. After the definitive surgery, serum creatinine level decreased from $1.43 \mathrm{mg} / \mathrm{dL}$ to $0.88 \mathrm{mg} / \mathrm{dL}$ within postoperative day 3. Follow-up abdominal CT on postoperative day 8 demonstrated a well-reconstructed right kidney with normal parenchymal enhancement without significant fluid collection (Fig. 3). He was discharged without any abdomen-related complications on postoperative day 20 .

\section{DISCUSSION}

Although the American Association for the Surgery of Trauma's injury scale was not designed as the treatment guideline, patients with low-grade renal injuries (grades I -III) are dominantly managed nonoperatively, whereas those with high-grade injuries (grades IV-V) have an increased likelihood of surgical intervention (1). Patients managed nonoperatively had a lower mortality rate, less likely required blood transfusion, and had shorter hospital stay compared with nephrectomy but similar to renorrhaphy (2). Renorrhaphy can be successfully performed to prevent unnecessary nephrectomy in grade IV renal injuries with hemodynamic instability.

Here, we described a successful renorrhaphy for grade IV renal laceration with unstable hemodynamics after blunt trauma. After controlling the renal pedicle using a vascular clamp (clamping time, $21 \mathrm{~min}$ ) following the opening of Gerota's fascia, the renal collecting system 
was closed using an absorbable suture to provide a watertight seal, and multifocal lacerations in the renal parenchyma were also sutured using pledgeted horizontal mattress sutures. An early decision regarding renal salvageability should be made, and the magnitude of the required reconstructive effort to repair the injury should be determined once hemorrhage is controlled.

\section{Conflicts of Interest Statement}

None of authors have a conflict of interest.

\section{REFERENCE}

1. Santucci RA, Fisher MB. The literature increasingly supports expectant (conservative) management of renal trauma: a systematic review. J Trauma. 2005;59:493 - 503.

2. Bjurlin MA, Jeng EI, Goble SM, Doherty JC, Merlotti GJ. Comparison of nonoperative management with renorrhaphy and nephrectomy in penetrating renal injuries. J Trauma. 2011;71(3):554-8.

\section{Video Legend}

Video 1. A video recording shows renorrhaphy with pledgeted sutures during clamping the renal vascular pedicle for grade IV renal injury. 